\title{
RING-SHAPED PIEZOELECTRIC MICROMACHINED ULTRASONIC TRANSDUCERS (PMUT) WITH INCREASED PRESSURE GENERATION
}

\author{
B.E. Eovino ${ }^{1 *}$, S. Akhbari ${ }^{1}$, and L. Lin $^{l}$ \\ ${ }^{1}$ University of California, Berkeley, California, USA
}

\begin{abstract}
As the application space of piezoelectric micromachined ultrasonic transducers (pMUT) expands and enters the industrial realm, it is necessary to continue improving the performance of individual devices, two of the most important metrics of which are generated acoustic pressure magnitude and directivity. Here, we present the first ring-shaped pMUT (r-pMUT), which have been fabricated using CMOS-compatible aluminum nitride (AIN) as both the transduction and elastic material. The r-pMUT exhibit newfound design freedom compared to standard circular pMUT (cpMUT), as their resonance frequency is found to be insensitive to mean radius $r_{0}$. The frequency responses of fabricated devices were measured and shown to match well with finite element model (FEM) simulations. By comparing r- and c-pMUT designed to operate at $1.5 \mathrm{MHz}$ with the same film stack, we show that $\mathrm{r}$ pMUT are capable of generating over $11.8 \times$ the pressure and with over $9 \times$ the directionality of their circular counterpart. As such, this device architecture could allow for highly efficient pMUT in applications that benefit from high pressures and directionality, such as rangefinding, fingerprint scanning, and therapeutics.
\end{abstract}

\section{INTRODUCTION}

Piezoelectric micromachined ultrasonic transducers (pMUT) are receiving great levels of interest as a technology that can be integrated with consumer electronics or in the medical field, with a growing application space that includes fingerprint scanning, imaging, gesture recognition, and therapeutics [1]-[4]. Typical pMUT operation includes both the launching and receiving of ultrasonic acoustic waves, making the pressure generated per volt one of the most critical performance metrics for pMUT. Therefore, improving the response of pMUT has been the topic of a myriad of recently reported design innovations [4]-[8]. For example, the dual-electrode bimorph pMUT shows a response that is $4 \times$ that of the state of the art [4]. Curved aluminum nitride (AIN) diaphragms show a static displacement that is $50 \times$ higher than flat devices with the same diameter [5]. An impedance matching tube has been used to increase the sound pressure level (SPL) by 350\% [6], and concentric venting rings are shown to increase the SPL by $67 \%$ [7]. Conversely, a forward-looking array of concentric ring-shaped capacitive MUT (cMUT) was used to generate large pressures at an acoustic focal point while minimizing the number of drive channels [8], however, a thorough study of the individual transducers that comprise the array has not been reported.

In this work, we introduce the first ring-shaped pMUT ( $\mathrm{r}-$ pMUT) and study the ring-shaped MUT as a standalone structure. At the cost of very little added fabrication complexity, r-pMUT are shown to have significantly increased design freedom compared to conventional circular pMUT (c-pMUT) by allowing for the same resonance frequency to be achieved with different topographic geometries. Furthermore, finite element method (FEM) simulations are developed and used to evaluate the performance of the r-pMUT by way of comparison with a c-pMUT designed with the same film stack and resonance frequency. The r-pMUT shows a $1080 \%$ greater SPL, which is the largest reported improvement over the state of the art. Additionally, the r-pMUT displays a directivity factor that is $8.3 \times$ larger than that of the c-pMUT.

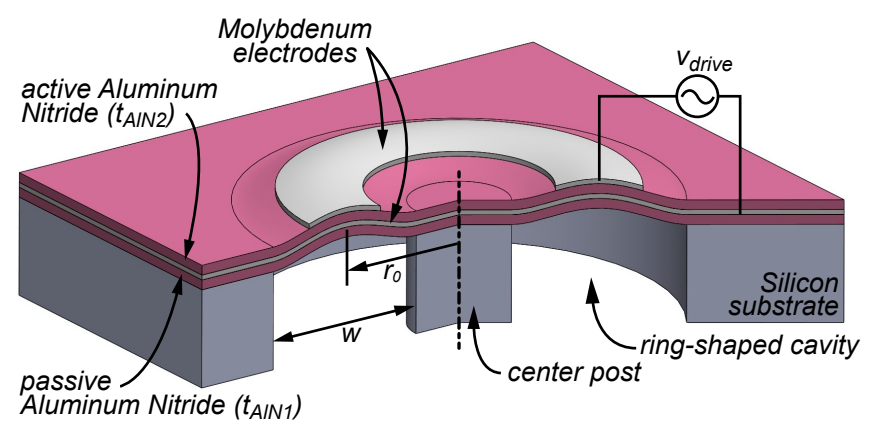

Figure 1: Cross-section schematic drawing of a ring-shaped pMUT being driven by an applied voltage.

\section{RING-SHAPED PMUT DESIGN \\ Concept}

A conceptual cross-sectional schematic of the (deformed) rpMUT is shown in Fig. 1, along with the design variables which define the diaphragm: mean radius $r_{0}$ and width $w$. Conversely, diaphragms in c-pMUT are defined only by the clamped boundary radius $a$. The operating principle is similar to that of a standard cpMUT: stress is induced in the active AlN layer when a voltage is applied between the top and bottom electrodes due to the $d_{31}$ piezoelectric effect, which in turn generates a bending moment and deforms the diaphragm in an axisymmetric flexural mode as shown. In contrast to the c-pMUT which has only one clamped boundary, the r-pMUT has two clamped boundaries at $r_{0} \pm w / 2$, since both the center post and substrate ideally act as mechanical anchors. As a reference to the reader, it was found throughout this study that in order to design r- and c-pMUT with the same film stack and equal resonance frequencies, $w \approx 1.48 a$.

\section{Fabrication}

As the fabrication of the r-pMUT has no added complexity compared to the c-pMUT, the process used in this work nearly exactly follows the CMOS- compatible process used previously by our lab for c-pMUT [9], and is shown as a process flow in Fig. 2. The fabrication begins with (a), the sputter deposition of the AlN $(1000 \mathrm{~nm}) / \mathrm{Mo}(130 \mathrm{~nm}) / \mathrm{AlN}(800 \mathrm{~nm}) / \mathrm{Mo}(130 \mathrm{~nm})$ film stack, where the $t_{A I N 1}>t_{A I N 2}$ to ensure that the neutral axis of the diaphragm is not inside of the active layer to prevent performance reduction [10]. In (b), the top electrode is patterned by a $\mathrm{SF}_{6}$ plasma etch, then the ground vias are patterned by first depositing $500 \mathrm{~nm}$ of PECVD $\mathrm{SiO}_{2}$ as a hardmask, followed by RIE of $\mathrm{SiO}_{2}$ and a two-step etch of AlN using first a Chlorine-based plasma and a subsequent wet etch in MF-319. The top electrode is designed to have a $55 \%$ areal diaphragm coverage and mean radius of $r_{0}$ (i.e. is centered on the released diaphragm) as preliminary simulations indicate that this results in good coupling to the fundamental vibration mode of the diaphragm. The diaphragms are released (c) with a backside DRIE, and the devices are diced and mounted on a custom PCB (d) using thermal release tape and silicone adhesive to ensure a full seal. 

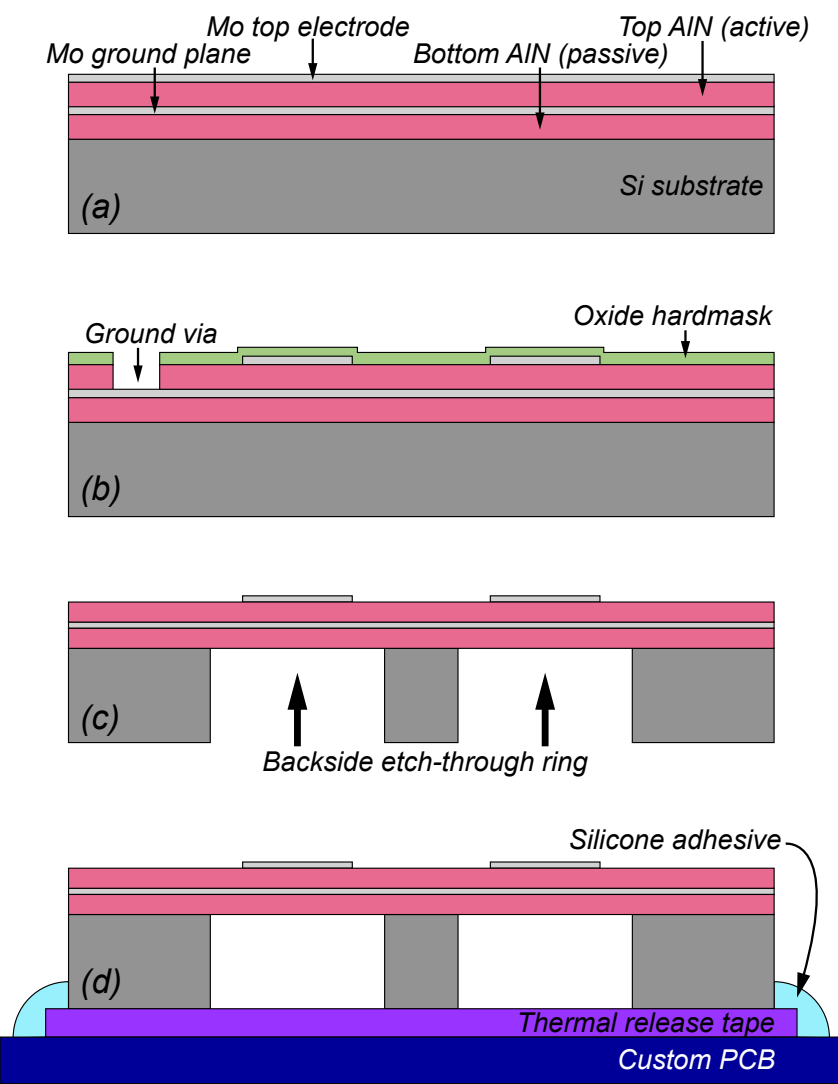

Figure 2: Fabrication process flow for the r-pMUT: (a) sputter film stack deposition, (b) top electrode patterning, hardmask deposition, and via etching (c) backside DRIE release etch (d) mounting to custom PCB for testing
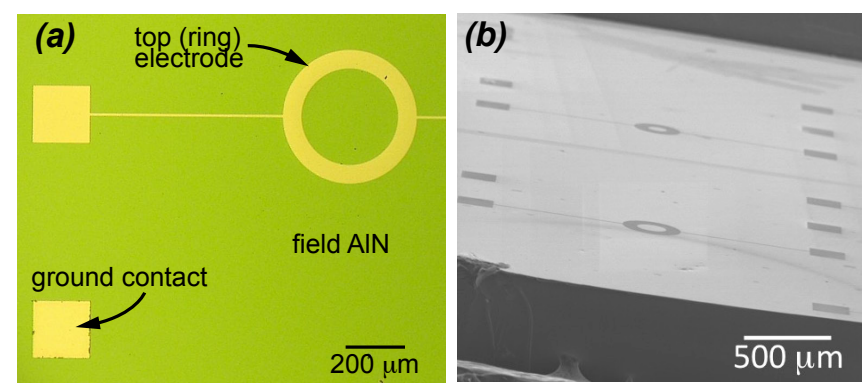

(c) over-vertical
sidewalls during mounting)
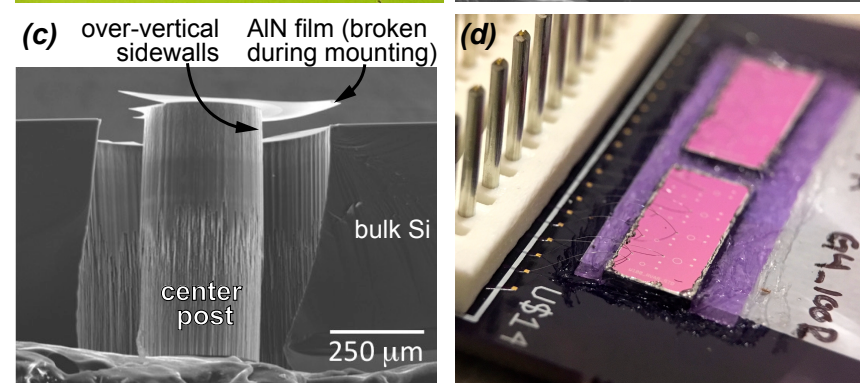

Figure 3: (a) optical micrograph of a r-pMUT before mounting, (b) and (c) oblique and cross-sectional SEM micrographs, respectively, (d) optical image of the r-pMUT mounted on a custom PCB for testing.

Fig. $3 \mathrm{a}$ and $3 \mathrm{~b}$ respectively show optical-top and SEMoblique views of the released r-pMUT (after completion of step (c) in Fig. 2), showing that the ground vias are fully exposed without
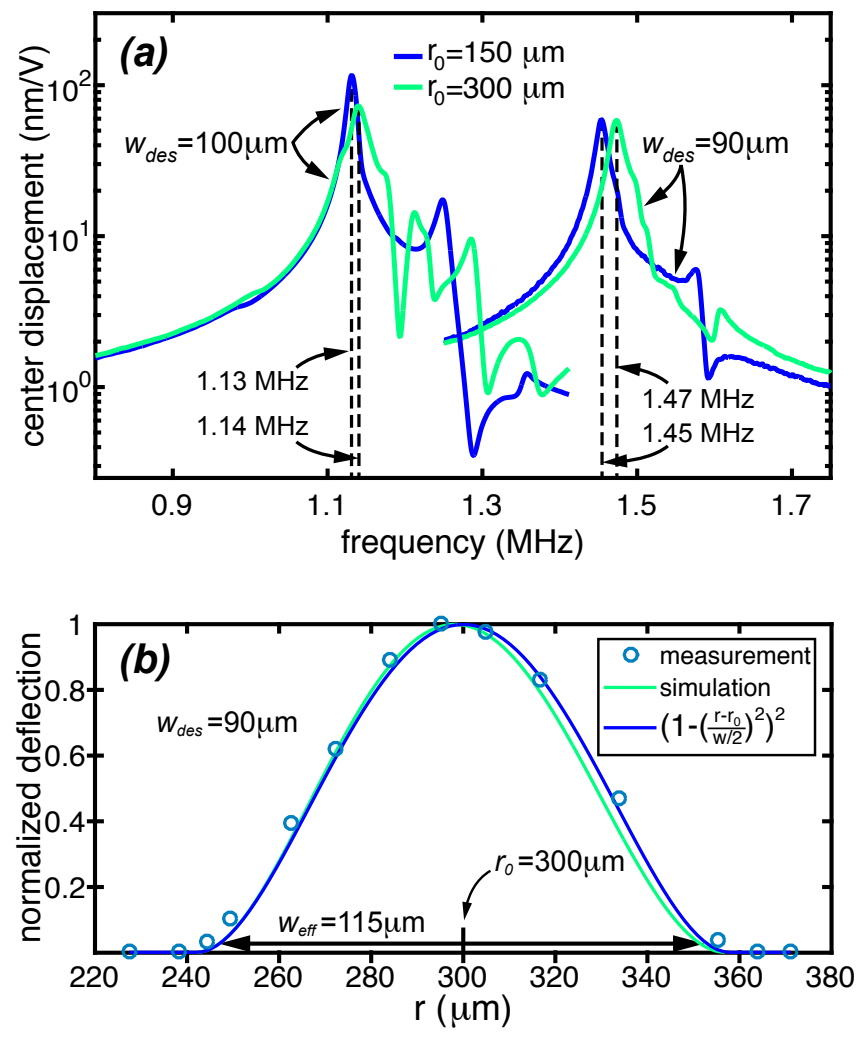

Figure 4: (a) measured displacement frequency responses of four $r$-pMUT showing the small dependence of resonance frequency on $r_{0}$, and strong dependence of resonance frequency on $w$. (b) Measured, simulated, and analytical vibration mode shape, displaying excellent agreement and ensuring that the r-pMUT $\left(w_{\text {des }}=90 \mu \mathrm{m}\right.$ and $\left.r_{0}=300 \mu \mathrm{m}\right)$ vibrates in the intended mode.

any residual AlN or damage to the Mo ground plane. Fig. $3 \mathrm{c}$ shows a cross-section SEM of a r-pMUT that broke while mounting for imaging. During the DRIE backside release process, issues with silicon undercutting result in larger fabricated diaphragm widths than the designed values. This effect is most pronounced along the center post, and was found to result in a 20$30 \mu \mathrm{m}$ discrepancy between the designed and actual width by using confocal microscopy (LEXT OLS3000, Olympus) for sidewall profile measurements. For this reason the designed and effective widths will henceforth be referred to using the variables $w_{\text {des }}$ and $w_{\text {eff }}$, respectively. Methods of mitigating this phenomenon are currently being explored. Fig. 3d presents an optical image of the fabricated devices attached and wire-bonded to a custom PCB for testing.

\section{RESULTS}

\section{Device Characterization}

The fabricated r-pMUT were characterized with a laser Doppler vibrometer (LDV, OFV-5000, Polytec Inc.) in air. Fig. 4a displays the results of four typical displacement frequency responses for r-pMUT with center radii $r_{0}=150$ (blue) and 300 (green) $\mu \mathrm{m}$, and designed widths $w_{d e s}=90$ and $100 \mu \mathrm{m}$. While high frequency spurious resonances are observed and are likely due to fabrication imperfections, the fundamental resonance frequencies for r-pMUT with $w_{d e s}=90$ and $100 \mu \mathrm{m}$ varied from $1.45-1.47 \mathrm{MHz}$ and 1.13-1.14 MHz respectively, both of which represent a less 
than $1.5 \%$ deviation as $r_{0}$ is doubled. As there is only one possible geometry for a given film stack and operational frequency with cpMUT, this insensitivity of resonance frequency to $r_{0}$ allows for an extra degree of design freedom in r-pMUT and can be explained by considering the equation for resonance frequency:

$$
f_{0}=\frac{1}{2 \pi} \sqrt{\frac{k}{m}}
$$

where $k$ and $m$ are the modal mass and stiffness of the structure. It is intuitive that mass is proportional to surface area $A$, and we can approximately say that $m \propto r_{0}$ after considering that $A \approx 2 \pi r_{0} w$, however, the relationship between $k$ and $r_{0}$ is less intuitive. A firstorder approximation can be derived for the case of $r_{0} \gg w$ by conceptually "unwrapping" the r-pMUT into a long rectangular strip with length $L=2 \pi r_{0}$ and width $w$, anchored along the two long boundaries and free along the two short boundaries. By definition $k=F / x$, where $x$ is the static deflection, and it follows that

$$
k=\frac{F}{x}=\frac{P A}{x}=\frac{P L w}{x}
$$

where $P$ is a uniform pressure applied on the diaphragm. Since the static deflection of a rectangular diaphragm with these boundary conditions does not depend on the length of the diaphragm, the only variable in Eq. 2 that depends on $r_{0}$ is $L$, so it can be approximated that $k \propto r_{0}$. Therefore, $r_{0}$ cancels out of Eq. 1 , and $f_{0}$ should show no dependence on the mean radius.

In order to ensure that the devices operate in the assumed mode, the vibration mode shape was additionally measured by recording the vibration amplitude around the resonance frequency and at different radial locations of the device. The results of such measurements taken at $1.4 \mathrm{MHz}$ for an r-pMUT with $w_{d e s}=90 \mu \mathrm{m}$ and $r_{0}=300 \mu \mathrm{m}$ are displayed Fig. $4 \mathrm{~b}$, alongside comparisons with a simplified finite element method (FEM) simulation by COMSOL Multiphysics, and an analytical approximation which is given by

$$
\phi(r)=\left(1-\bar{r}^{2}\right)^{2}
$$

where $\phi(r)$ is the mode shape, and $\bar{r}=2\left(r-r_{0}\right) / w_{\text {eff }}$ is a normalized radial coordinate that varies from -1 to 1 at the inner and outer

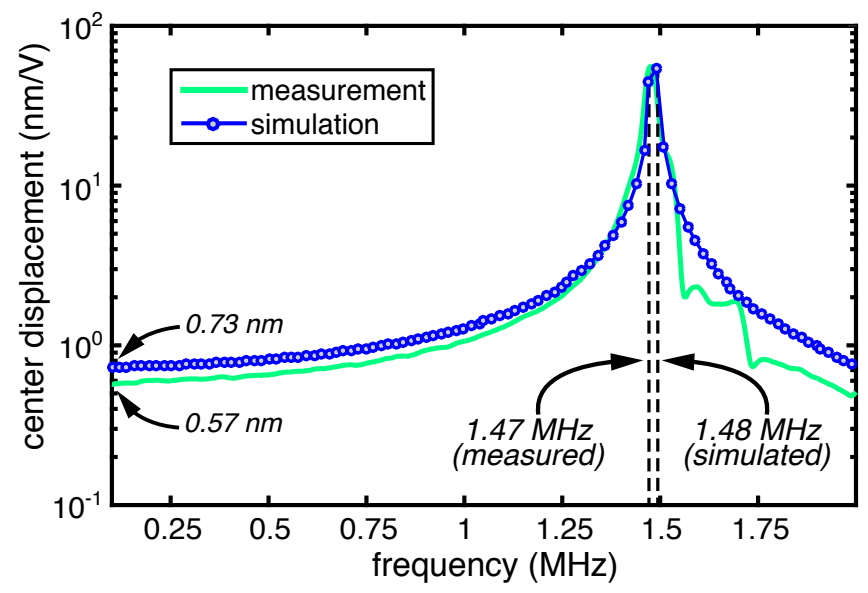

Figure 5: Comparison of measured and simulated displacement frequency responses for r-pMUT, showing good agreement between the low-frequency displacement and resonance frequency. clamped boundaries, respectively. All three methods show excellent agreement, and as expected, the center post and bulksubstrate act as anchors and the peak displacement occurs near $r_{0}$. Furthermore, an effective width of $w_{\text {eff }}=115 \mu \mathrm{m}$ is observed, which is consistent with the aforementioned fabrication errors.

In order to further validate the FEM model and ensure its ability to correctly predict device performance, frequency response simulations were also compared with LDV measurements. Fig. 5 shows one such comparison for an r-pMUT with $w_{d e s}=90 \mu \mathrm{m}$, $w_{\text {eff }}=115 \mu \mathrm{m}$, and $r_{0}=225 \mu \mathrm{m}$ where an excellent agreement between model and experiment is displayed by a close match between measured and simulated values for both (1) low-frequency displacement ( 0.57 and $0.73 \mathrm{~nm}$, respectively) and (2) resonance frequencies (1.47 and $1.48 \mathrm{MHz}$, respectively).

\section{Acoustic Performance}

To compare the acoustic performance of $r-$ and c-pMUT, FEM simulations of two prototype devices (ring: $w=115 \mu \mathrm{m}$, $r_{0}=225 \mu \mathrm{m}, 55 \%$ electrode coverage, and circle: $a=77.25 \mu \mathrm{m}, 70 \%$ electrode coverage) designed for $1.5 \mathrm{MHz}$ operation with $800 / 1000$ $\mathrm{nm}$ thick AlN film stacks were performed. Fig. 6a and $6 \mathrm{~b}$ respectively show the peak displacement and far-field pressure for the prototype transducers. Despite having a peak displacement of only $186 \mathrm{~nm} / \mathrm{V}$ compared to $298 \mathrm{~nm} / \mathrm{V}$ for the c-pMUT, the rpMUT displays a peak pressure of $201 \mathrm{~Pa}$, which is more than $7.7 \times$ greater than the $23 \mathrm{~Pa}$ generated by its circular counterpart. The r-pMUT is able to generate such high acoustic pressures because $P \propto V_{v}$, where $V_{v}=v_{\text {avg }} A$ is the volume velocity and $v_{\text {avg }}$ is the average diaphragm velocity, and the radiating area of an $r$ pMUT may be made much larger than that of the c-pMUT by choice of $r_{0}$. Indeed, as shown in Fig. $6 \mathrm{c}$, the pressure generated by the r-pMUT shows a strong dependence on $r_{0}$, and reaches a value of $272 \mathrm{~Pa}$ when $r_{0}=325 \mu \mathrm{m}$, over $11.8 \times$ that of the prototype c-pMUT. The simulated $1080 \%$ increase in SPL represents the largest reported improvement resulting from a design change. At very large $r_{0}$ the pressure begins to decrease due to increased mass loading from the acoustic medium and the effects of sidelobes. Fig. $6 \mathrm{~d}$ shows the normalized far-field pressure from the ring and circle prototypes. Owing to its larger acoustic aperture, the prototype ring has a more directional acoustic output, displaying a directivity factor, which is the ratio of peak to average acoustic intensity, of 19 compared to 2 for the circle and thereby improving efficiency.

\section{CONCLUSION}

We have introduced, experimentally characterized, and performed FEM simulations on the first-ever ring-shaped pMUT (r-pMUT). An insensitivity of the resonance frequency on the mean radius has been observed, allowing r-pMUT an extra degree of design freedom compared to circular pMUT (c-pMUT), as the topographic geometry is no longer constrained by the resonance frequency. Owing to this fact, r-pMUT can be designed with a larger volume velocity than c-pMUT, thereby increasing their pressure generation. In fact, by comparing $1.5 \mathrm{MHz}$ devices made of the same film stack, r-pMUT show an estimated $1080 \%$ increase in SPL and $830 \%$ increase in directivity over c-pMUT. These factors indicate that r-pMUT may be ideal for forward-looking applications that require large acoustic pressures, such as rangefinding, fingerprint scanning, and therapeutics. Further benefits may also be observed by creating concentric arrays of $r-$ pMUT, or even incorporating a concentric c-pMUT, and should be explored in the future. 

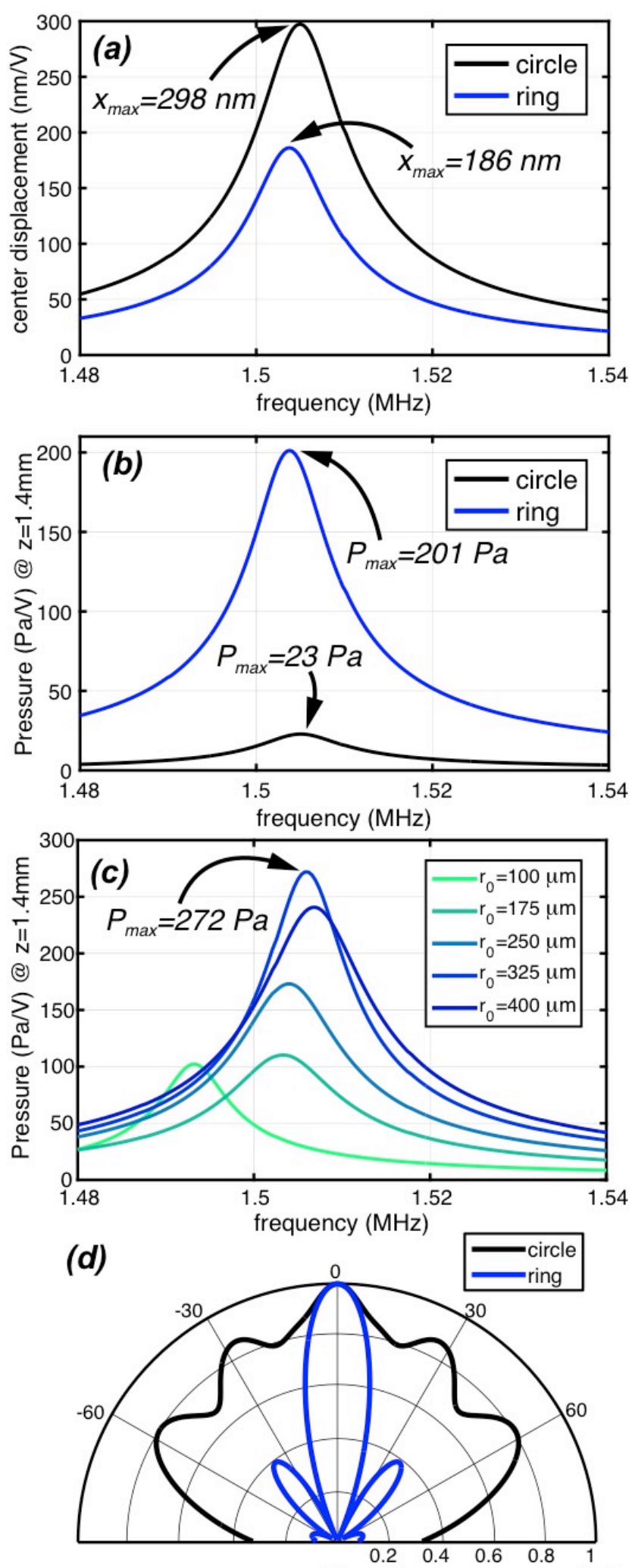

Normalized pressure amplitude

Figure 6: Simulated (a) displacement and (b) far-field pressure frequency response near resonance of prototype transducers, (c) far-field pressure for $r-p M U T$ with $w=115 \mu \mathrm{m}$ and various $r_{0}$, (d) normalized pressure amplitude of prototype transducers as a function of angle.

\section{ACKNOWLEDGEMENTS}

This work was supported in part by National Science Foundation Graduate Research Fellowship Program under Grant No. DGE 1106400. The authors would also like to thank Xiaoyue Jiang and Levent Beker for helpful discussions, members of Prof. David Horsley's lab from UC Davis for assistance with LDV measurements, and the Transducer Research Foundation for generously providing travel support.

\section{REFERENCES}

[1] X. Jiang, H. Tang, Y. Lu, X. Li, J. Tsai, E. Ng, M. Daneman, M. Lim, F. Assaderaghi, B.E. Boser, and D.A. Horsley, "Monolithic 591x438 DPI ultrasonic fingerprint sensor." in 2016 IEEE 29th International Conference on Micro Electro Mechanical Systems (MEMS), pp. 145-148, Shanghai, China

[2] D.A. Horsley, R.J. Przybyla, M.H. Kline, S.E. Shelton,A. Guedes, O. Izyumin, and B.E. Boser, "Piezoelectric micromachined ultrasonic transducers in consumer electronics: The next little thing?" in 2016 IEEE 29th International Conference on Micro Electro Mechanical Systems (MEMS), pp. 145-148, Shanghai, China

[3] R.J. Przybyla, H.Y. Tang, S.E. Shelton, D.A. Horsley, and B.E. Boser, "3D ultrasonic gesture recognition," in Technical Digest of the 2014 IEEE International Solid-State Circuits Conference (ISSCC), pp. 210-211, San Francisco, California.

[4] S. Akhbari, F. Sammoura, B.E. Eovino, C. Yang, and L. Lin, "Bimorph Piezoelectric Micromachined Ultrasonic Transducers," Journal of Microelectromechanical Systems, advanced online publication, DOI 10.1109/JMEMS2016.2516510

[5] S. Akhbari, F. Sammoura, S.E. Shelton, C. Yang, D.A. Horsley, and L. Lin, "Highly responsive curved aluminum nitride pMUT," in 2014 IEEE 27th International Conference on Micro Electro Mechanical Systems (MEMS), pp. 124-127, San Francisco, California

[6] S.E. Shelton, O. Rozen, A. Guedes, R.J. Przybyla, B.E. Boser, and D.A. Horsley, "Improved acoustic coupling of aircoupled micromachined ultrasonic transducers," in 2014 IEEE 27th International Conference on Micro Electro Mechanical Systems (MEMS), pp. 124-127, San Francisco, California

[7] O. Rozen, S.T. Block, S.E. Shelton, and D.A. Horsley, "Piezoelectric micromachined ultrasonic transducer with increased output pressure via concentric venting rings," in Proc. Solid-State Sensors, Actuators and Microsystems Conference (TRANSDUCERS) 2015, pp. 670-673, Anchorage, Alaska

[8] S.H Wong, M. Kupnik, R.D. Watkins, K. Butts-Pauly, and B.T. Khuri-Yakub, "Capacitive micromachined ultrasonic transducers for therapeutic ultrasound applications," in IEEE Transactions on Biomedical Engineering, vol. 57, no. 1, pp. 114-123, 2010.

[9] S. Akhbari, F. Sammoura, C. Yang, M. Mahmoud, N. Aqab, and L. Lin, "Bimorph pMUT with dual electrodes," in 2015 28th IEEE International Conference on Micro Electro Mechanical Systems (MEMS), pp. 928-931, Estoril, Portugal

[10] P. Muralt, N. Ledermann, J. Baborowski, A. Barzegar, S. Gentil, B. Belgacem, S. Petitgrand, A. Bosseboeuf, and N. Setter, "Piezoelectric micromachined ultrasonic transducers based on PZT thin films," IEEE Transactions on Ultrasonics, Ferroelectrics, and Frequency Control, vol. 52, no. 12, pp. 2276-2288, 2005.

\section{CONTACT}

*B. Eovino, tel: +1-408-410-7375; beovino@berkeley.edu 